\title{
Evaluation of Subjective Response to Lighting
}

\author{
Belinda L. Collins
}

National Institute of Standards and Technology

Gaithersburg, Maryland 20899

\section{SUMMARY}

This paper summarizes findings from a more extensive review of the research literature and from NIST research on the subjective response to lighting and luminance distributions. It suggests strongly that luminance distributions and patterns play an important role in determining positive psychological response to lighting.

\section{INTRODUCTION}

The primary goal of research into the psychological effects of lighting is to determine if there are reliable, repeatable subjective responses to different lighting environments. The terms psychological and subjective refer to emotional or affective responses to lighting - both positive and negative - as distinguished from visual or task performance. In a longer review, Collins (1993) discussed research into the psychological response to lighting both from laboratory and field research. Her findings are summarized briefly here.

\section{BACKGROUND}

The research literature supports the existence of general, albeit imprecise, models which link psychological response to different physical dimensions of lighting. Flynn and his coworkers in the United States identified important dimensions of lighting which could be reliably related to physical parameters such as luminance patterns and distributions within the environment. Loe and his colleagues in the U.K. validated Flynn's results while extending the measurement of luminance distributions. The British research complemented Flynn's research in the U.S. while reiterating that lighting moods and perceptions such as bright/dim, uniform/non-uniform, and overhead/peripheral could be reliably elicited by specific variations in the luminance distributions. Additionally, Shepherd and her colleagues at the University of Sydney identified important physical parameters related to the perception of gloom which indicated that lighting patterns can have a negative as well as a positive effect. Recent research in Japan by Inui and his colleagues has suggested that artificial luminance distributions tend to have much higher contrasts as well as greater spatial distribution of high contrasts than natural scenes. These researchers also identified physical variables which are responsible for the perception of spaciousness within a room. Boyce and Cuttle (1990) found that the spectral power distribution of a light source plays a critical role in determining the clarity of a visual scene as well as the accuracy of the perception of color appearance. Finally, Collins and others reinforced the importance of luminance patterns in determining satisfaction with actual office environments. Thus, the analysis of research into the subjective response to lighted environments reveals that psychological response is linked to the amount and placement of light within a space, as well as to its spectral characteristics.

\section{LABORATORY RESEARCH}

Flynn et al. $(1978,1979)$ found that perceptions of clarity are associated with central overhead lighting, while the perception of spaciousness is related to the use of peripheral and wall washing systems. Poor visual clarity and dissatisfaction with the lighted environment are observed with 
extremely non-uniform lighting designs. While the underlying dimensions of psychological response were identified and associated with particular lighting designs, no indication was given as to the magnitude of the effects or the limits at which a configuration switched from peripheral to overhead or from bright to dim.

Inui and Miyata $(1973,1977)$ and Sato et al. (1989) found that the perception of spaciousness increases as the horizontal illuminance increases, as well as percentages of window area, room volume, and sky luminance. They suggested that although there may be a lower bound to just acceptable spaciousness, there does not appear to be an upper bound (in which a space is too spacious to be acceptable). Increasing horizontal illuminance, providing windows, and increasing room volume all increase the perception of spaciousness both in scale models and in actual rooms. Furthermore, spaces that appear to be more spacious are considered to be more friendly, with no apparent upper limit on spaciousness.

Although different lighting designs have been shown to elicit positive subjective reactions, Shepherd et al. (1990) found that variations in lighting configurations can result in impressions of gloom, as well as pleasantness, in a space. The research suggests that impressions of gloom arise from inadequate or uneven distribution of light in a room such that the details in the corners and periphery are not discriminable. Overall light levels which are in the mesopic range as well as low surface reflectances also contribute to the impression of gloom.

Other researchers have examined the response to different luminance patterns and distributions in simulated offices in an attempt to set desired ratios between task and immediate surround and/or vertical and horizontal surfaces. To this end, Hentschel (1990) and Van Ooyen et al. (1986) defined suggested luminance ratios for desk, immediate surround, walls, and ceiling which are likely to produce positive psychological effects. In addition, the review of the research demonstrates that variations in luminance distribution have a marked effect on the overall appearance of space both in terms of brightness and interest. Thus, scenes that are considered bright tend to have high surface luminances (above $100 \mathrm{~cd} / \mathrm{m}^{2}$ ) in the central field of view, rather than simply having luminaires with high luminances. There is a point beyond which brightness becomes excessive, however, such that luminances above $800 \mathrm{~cd} / \mathrm{m}^{2}$ begin to be considered glaring rather than bright. Scenes that are considered interesting have variations in the brightness pattern (discussed in terms of the ratio of maximum to minimum luminances) with non-uniform lighting distributions in the central 40 degree field of view (Loe et al., 1991). Inui and Nakamura (1987) suggested that examination of luminance contours and frequency distributions of contrast could be a valuable means for evaluating the subjective response to luminance distributions.

\section{FIELD EVALUATIONS}

The studies reviewed up to this point have been laboratory studies in which good control could be maintained over the lighting variables. It is not known whether findings from laboratory studies would apply to actual offices, however. To assess the response to existing lighting situations, PostOccupancy Evaluations (POE) have been used to measure both psychological response and physical characteristics of workspaces after initial occupancy or retrofit. NIST has conducted POE studies in more than 20 buildings. These surveys have indicated the importance of good lighting in achieving satisfaction with a work environment, as well as the ability to control lighting, particularly task lighting to minimize glare and reflections from overhead lighting for VDT users.

In an extensive POE study of 912 work stations in thirteen buildings, Collins et al. . (1989 a,b), and Collins (1990) evaluated occupant satisfaction with their lighting and reaction to different design and illumination parameters. Although seven different lighting systems were identified in these work stations, the analysis discussed here focused on a subset of three systems - one, a direct system; two, 
a direct system with fixed furniture mounted task lighting; and three, an indirect system with the same fixed furniture mounted task lighting. In the study, occupants rated their satisfaction with the lighting, as well as their perceptions of the brightness of the work spaces.

Analysis of the data revealed that lighting satisfaction was consistently lower for work stations with furniture integrated lighting. Furthermore, rated satisfaction decreased with increasing illuminance for people at work stations with furniture integrated task lighting. Conversely, occupant satisfaction was significantly higher for work stations without task lighting, and showed a very slight tendency to increase with illuminance. For all work stations, task illuminances were generally within the range (200-1000 lux) recommended by the IESNA for performance of visual tasks of medium to high contrast or small to medium size. Finally, people in work stations that had some daylight availability were significantly more satisfied than those without any daylight.

As noted above, two ambient lighting systems - direct and indirect - were used at a substantial number of work stations with identical systems furniture and furniture integrated lighting. This meant that the effects of the type of ambient lighting on lighting satisfaction and perceived brightness could be explored for a common task-lighting configuration. Comparison of the responses to the two lighting systems revealed that 68 percent of those with a direct ambient system were satisfied with their lighting, as compared with only 54 percent of those with an indirect ambient system. Thus, the combination of indirect ambient lighting with integrated task lighting was particularly unsuccessful.

Furthermore, although the mean primary task illuminance was higher for fixed task lighting with indirect ambient lighting, nearly half (43 percent) of the occupants with this configuration rated their work station as being dim as compared to 21 percent of those with the direct configurations. In addition, over two-thirds (68 percent) of the occupants with the combined indirect ambient-fixed task configuration rated the amount of light available for work as being low, even though the illuminances directly at the primary work station were actually the highest of the seven systems identified. Subjective brightness was clearly an important contributor to perceived lighting quality: no space that was perceived as dim was considered to have the right amount of light for work. These data suggest that the perceived brightness of a work station was an important determinant of the occupant's perception of the lighting quality within the space.

Although ten measures of luminance were obtained, no single measure could be related to lighting satisfaction ratings in any meaningful way. As a result, the ten measures were averaged to explore the relation between lighting satisfaction and luminance patterns in the space. The rationale for averaging all the luminances is based on the hypothesis that the occupants' response to the brightness of their spaces is a response to the whole space - to its total lighting gestalt - as opposed to a single luminance value. This analysis indicated that the majority of work stations (68 percent) with the indirect system and task lighting had a very low average luminance - between $0-167 \mathrm{~cd} / \mathrm{m}^{2}$ - as compared with 14 percent of those with the direct system with task lighting.

Examination of occupant ratings of work station brightness found that these could be predicted more by average luminance than to task illuminance, particularly for work stations with furniture integrated lighting. Occupants rated spaces with lower average luminances as dim, while rating those with higher average luminances as bright. Furthermore, the data indicated that the relationship between subjective brightness and average room luminance was stronger than that between brightness and task illuminance, particularly for indirect/task lighting systems, suggesting strongly that occupants based their judgements of brightness on room luminance rather than task illuminance.

Comparison of the mean ratings for work station lighting satisfaction revealed that the highest ratings were given for the direct system without task lighting. They were also greater than those for the same system with task lighting, while those for the indirect system were always lower than either of 
the two direct systems. There was a similar relationship between the three systems for the brightness ratings. The combined indirect ambient-integrated task configuration had an overall average luminance that was less than half of that for each of the other systems. The means for the luminance of the ceiling and brightest area in the field of view were also substantially below those for the two direct systems. Yet, task illuminance (and luminance) was consistently higher. Overall lighting satisfaction and brightness ratings were consistently lower for the indirect/task system than for the other two systems.

Collins et al. found that many of the negative responses to indirect/task lighting could be related to the combination of a fixed task lighting system with an indirect ambient system. Unlike many task lighting systems, this particular one was not adjustable. It was located at the back of the desk, concealed under an upper shelf, so that light was directed toward the task and could easily cause specular reflections on the task, thereby reducing task contrast and visibility. Occupants had no control over the position or direction of the task light. In addition, the ambient lighting which was located in the top of the furniture system and directed up to the ceiling often resulted in a non-uniform pattern of ceiling luminance with many dark areas. Furthermore, the room luminances were much lower than for any other system, while the mean task illuminances were higher. The result appears to have been a most unsatisfactory lighting system for many occupants. By contrast, a direct configuration was rated much more favorably, even with the same type of systems furniture. In fact, the variable most strongly related to work station lighting satisfaction was work station brightness, which was perceived to be lowest for the combination of indirect ambient with integrated task lighting. The analysis suggests that these spaces were characterized by extremes - with very bright tasks and very dim surroundings. As a result, it appears that the pattern of luminances in the space was a more important factor in influencing occupants' satisfaction with their lighting and perceptions of brightness in their spaces than the amount of light on the task. Collins et al. suggested that the combination of a bright task surface with dim surroundings produced an unappealing lighting situation. These considerations appeared to be even more important than the amount of light on the task for determining satisfaction with lighting.

\section{CONCLUSIONS}

The preceding discussion highlights the importance of luminance distributions in determining psychological response to lighting and suggests that the distribution of light plays a critical role in determining whether a space appears pleasant or gloomy, satisfying or unsatisfying, spacious or cramped. Use of high color rendering lamps can reinforce the positive emotional response created by a luminance distribution. Moderate variation in the lighting distribution over space and time is also associated with a positive response. Thus, lighting can be used to elicit positive or negative response to an environment - findings which occur in both laboratory and field studies. The research literature reveals considerable evidence that people find certain lighted environments to be both pleasant and satisfying. There is ample indication that the pattern of luminance in a space has a powerful impact on its pleasantness or gloominess. Most of the research data tends to be general rather than specific in providing directions for achieving a desired effect, however.

\section{FURTHER RESEARCH}

Yet, despite the trends observed among the many studies of psychological reaction to lighting, many questions remain about predicting the effects of a particular design or type of lighting system. It is very difficult to be certain that a given light level or type of lamp or luminaire will produce the desired psychological effect. Some of the important issues that remain unresolved involve the need to determine the parameters of the physical dimensions responsible for different psychological responses. Thus, there are few guidelines to indicate when a lighting system will be perceived as uniform or non-uniform, as clear or hazy, as pleasant or gloomy, or as bright or dim. There are also 
questions about the role of both task illuminance and room luminance patterns as well as of the levels and distributions of light that are required to achieve specific psychological responses. For example, when does pleasantly bright become glaring, distracting, or just too bright? The findings that gloom results at levels below $10 \mathrm{~cd} / \mathrm{m}^{2}$, particularly with uneven distributions of light, should be replicated by other researchers in other institutions and in field as well as laboratory situations. At the same time, much more research is needed on preferred lighting levels, including documentation of the illuminances that people choose when they are free to choose. What is the contribution of different types of light sources, including daylight, to preferred illuminance levels and luminance distributions? What is the role of luminance patterns as compared with task illuminance in determining perceived brightness? Finally, the review of the literature reinforces the need for much greater use of appropriate instrumentation and procedures for determining the physical conditions in a space so that the psychological response can be linked in a meaningful way. Thus in the future, we are likely to see a much greater use of luminance scanners, spectral measurements, and determination of wall/surface reflectances and colors. Despite improved instrumentation, the need for better control and measurement of both physical and psychological variables remains.

Although further, more precise research is needed, the data indicate very strongly that there are certain lighting configurations which will reliably elicit certain responses. Thus, increasing lighting level, either for task illuminance or surround luminance, is generally associated with increased perceived brightness and satisfaction (up to some unspecified upper limit). In contrast, decreasing light level is not associated with greater satisfaction and perceived brightness. Similarly, increasing variety and interest are associated with positive psychological response, as is increased spaciousness. An overview of the trends in these relationships suggests that while there may be optimum levels of the physical variables, there is never a negative relationship in which lower brightness, less variety, less spaciousness, or lower CRI results in a more positive psychological response.

\section{REFERENCES}

Boyce, P.R. and Cuttle, C. Effect of correlated color temperature on the perception of interiors and color discrimination performance. Lighting Research and Technology, 1990, 22, Pp. 19-36.

Collins, B.L. Evaluation of Subjective Response to Lighting Distributions: A Literature Review. National Institute of Standards and Technology, Gaithersburg, MD, NISTIR 5119, February 1993.

Collins, B.L. Evaluation of the Role of Luminance Distributions in Occupant Response to Lighting, Proceedings, National Lighting Conference, Cambridge, April 1990, Pp. 1-10.

Collins, B.L., Fisher, W.S., Gillette, G.L., and Marans, R.M. Second Level Post-Occupancy Evaluation Analysis, Journal of the Illuminating Engineering Society, 1990, 19, Pp. 21-44.

Flynn, J.E., Spencer, T.J., Martyniuk, O., and Hendrick. C. Interim study of procedures for investigating the effect of light on impression and behavior. Journal of the Illuminating Engineering Society, 1973, Pp. 87-94.

Flynn, J.E., Spencer, T.J., Martyniuk, O., and Hendrick. C. A guide to methodology procedures for measuring subjective impressions in lighting. Journal of the Illuminating Engineering Society, 1979, Pp. 95-110.

Flynn, J.E. and Subisak, G.J. A procedure for qualitative study of light level variations and system performance. Journal of the Illuminating Engineering Society, 1978, $\underline{8}$, Pp.28-35 
Hentschel, H.-J. Preferred luminance ranges for indoor lighting. Proceedings CIBSE National Lighting Conference, 1990, Pp. 128-136.

Inui, M. and Miyata, T. Spaciousness in interiors. Lighting Research and Technology, 1973, 5, Pp. 103-111.

Inui, M. and Miyata, T. Spaciousness, behavior and the visual environment. Journal of Light and the Visual Environment, 1977, 1, Pp. 59-63.

Inui, M. and Nakamura, Y. Luminance distribution in natural and artificial landscapes. CIE Proceedings, Venice, 1987, Pp. 178-181.

Loe, D.L., Mansfield, K.P., and Rowlands, E. Light patterns and their relevance to spatial appearance and the quality of the lit environment. CIE Proceedings - 22nd Session, Melbourne, Australia, 1991, Pp. 41-44.

Loe, D.L., Rowlands, E., and Watson, N.F. Preferred lighting conditions for the display of oil and watercolour paintings. Lighting Research and Technology, 1982, 14, 173-192.

Rowlands, E., Loe, D.L., McIntosh, R.M., and Mansfield, K.P. The effect of light patterns on subjective preference. Proceedings CIE 20th Session (Amsterdam), 1983.

Sato, M., Inui, M., Nakamura, Y., and Takeuchi, Y. Visual environment of a control room. Lighting Research and Technology, 1989, 23, 99-106.

Shepherd, A.J., Julian, W.G., and Purcell, A.T. Lightness, brightness, and gloom in interior lighting. Proceedings CIBSE National Lighting Conference, 1990, Pp. 73-86.

Van Ooyen, M.H.F, Weijgert, J.C.A., and Begemann, S.H.A. Luminance distribution as a basis for office lighting design. Proceedings: National Lighting Conference, 1986, Pp. 103-108. 